\title{
A phosphate starvation response gene (psr1-like) is present and expressed in Micromonas pusilla and other marine algae
}

\author{
Cara L. Fiore ${ }^{1,3, *}$, Harriet Alexander ${ }^{2,4}$, Melissa C. Kido Soule ${ }^{1}$, \\ Elizabeth B. Kujawinski ${ }^{1}$ \\ ${ }^{1}$ Department of Marine Chemistry and Geochemistry, Woods Hole Oceanographic Institution, Woods Hole, MA 02543, USA \\ ${ }^{2}$ MIT/WHOI Joint Program in Oceanography and Applied Ocean Sciences, Department of Biology, \\ Woods Hole Oceanographic Institution, Woods Hole, MA 02543, USA \\ ${ }^{3}$ Present address: Biology Department, Appalachian State University, Boone, NC 28608, USA \\ ${ }^{4}$ Present address: Department of Biology, Woods Hole Oceanographic Institution, Woods Hole, MA 02543, USA
}

\begin{abstract}
Phosphorus (P) limits primary production in regions of the surface ocean, and many plankton species exhibit specific physiological responses to P deficiency. The metabolic response of Micromonas pusilla, an ecologically relevant marine photoautotroph, to P deficiency was investigated using metabolomics and comparative genomics. The concentrations of some intracellular metabolites were elevated in the P-deficient cells (e.g. xanthine, inosine), and genes involved in the associated metabolic pathways shared a predicted conserved amino acid motif in the non-coding regions of each gene. The presence of the conserved motif suggests that these genes may be co-regulated, and the motif may constitute a regulatory element for binding a transcription factor, specifically that of Psr1 (phosphate starvation response). A putative phosphate starvation response gene ( $p s r 1$-like) was identified in M. pusilla with homology to well characterized $p s r 1 / p h r 1$ genes in algae and plants, respectively. This gene appears to be present and expressed in other marine algal taxa (e.g. Emiliania huxleyi) in field sites that are chronically P limited. Results from the present study have implications for understanding phytoplankton taxon-specific roles in mediating $\mathrm{P}$ cycling in the ocean.
\end{abstract}

KEY WORDS: Micromonas pusilla Phosphate stress response - Marine algae - Metabolomics · Dissolved organic matter $\cdot$ Biological oceanography

\section{INTRODUCTION}

Phosphorus (P) is a critical element for life, and is found in lipid membranes, genetic material, and energy storage compounds. Because most marine microorganisms preferentially take up $\mathrm{P}$ as inorganic phosphate $\left(\mathrm{PO}_{4}{ }^{3-}\right)$, concentrations of dissolved inorganic $\mathrm{P}$ are generally below $1 \mu \mathrm{M}$ in the surface ocean (Karl 2014), which limits phytoplankton productivity (Tyrrell 1999).

Marine phytoplankton respond to chronic P deficiency by investing resources in $\mathrm{P}$ uptake via inor-

${ }^{*}$ Corresponding author: fiorec@appstate.edu ganic and organic compounds (Chung et al. 2003, Lin et al. 2016, Sosa et al. 2019), remodeling cellular metabolism and structures (Berdalet et al. 1994, Van Mooy et al. 2009, Martin et al. 2011, Shemi et al. 2016, Cañavate et al. 2017, Kujawinski et al. 2017), and storing $\mathrm{P}$ (Dyhrman et al. 2012, Martin et al. 2014). Different mechanisms for enacting these responses have evolved in widely distributed and often sympatric phytoplankton taxa. For example, within the diatom Phaeodactylum tricornutum, proteomics indicated broadly depressed metabolic activity as a

() The authors 2021. Open Access under Creative Commons by Attribution Licence. Use, distribution and reproduction are unrestricted. Authors and original publication must be credited. 
response to $\mathrm{P}$ starvation (Feng et al. 2015). Specifically, energy metabolism, amino acid metabolism, nucleic acid metabolism, and photosynthesis were down-regulated, while protein degradation, lipid accumulation, and photorespiration were up-regulated (Feng et al. 2015). In contrast, the prymnesiophytes Prymnesium parvum and Emiliania huxleyi and the dinoflagellates Prorocentrum donghaiense and Amphidinium carterae maintained energy-generating processes (i.e. photosynthesis) and carbon fixation in response to P deficiency (Lai et al. 2011, Li et al. 2016, Rokitta et al. 2016, Shi et al. 2017). Interestingly, $P$. donghaiense increased nitrate assimilation under P deficiency (Shi et al. 2017), whereas E. huxleyi reduced nitrate uptake with a tight coupling between $\mathrm{P}$ and nitrogen $(\mathrm{N})$ pools (Rokitta et al. 2016). While the experimental designs differ slightly across studies, the aggregate results indicate the presence of both taxon-specific differences and crosstaxon similarities in the physiological response of phytoplankton to P deficiency (Rengefors et al. 2003, Lomas et al. 2004, Lin et al. 2016, Martiny et al. 2020). These strategies all minimize non-critical P utilization and maximize $\mathrm{P}$ uptake, and thus play important roles in structuring phytoplankton assemblages in the oceans (Dyhrman \& Ruttenberg 2006, Dyhrman et al. 2009, Martin et al. 2011, Rokitta et al. 2016, Guo et al. 2018).

The cosmopolitan picoeukaryotic groups Micromonas and Ostreococcus spp. are important marine primary producers (Li 1994). Micromonas spp. are of particular interest due to the abundance and wide distribution of organisms in this genus (Demory et al. 2017). Recent culture experiments with $M$. pusilla isolated from Svalbard (Hoppe et al. 2018) and $M$. pusilla Lac38 (Maat et al. 2014) also indicated that these strains are likely to be successful under conditions of increased acidification and low nutrient $(\mathrm{P})$ availability. This success may be due, in part, to higher efficiency of carbon sequestration for growth under high $p \mathrm{CO}_{2}$ conditions and/or the ability to remodel photosystem proteins under nutrient-limited conditions (Maat et al. 2014, Guo et al. 2018, Hoppe et al. 2018). Additionally, similar to other phytoplankton (Chung et al. 2003), M. pusilla may shift lipid composition to increase non-P-containing lipids (Maat et al. 2016, Guo et al. 2018), as well as up-regulate genes and proteins for inorganic and organic P transporters (Whitney \& Lomas 2016, Guo et al. 2018) and for polyphosphate accumulation (Bachy et al. 2018). However, some of the broader physiological response mechanisms of $M$. pusilla and other prasinophytes to $\mathrm{P}$ deficiency have yet to be fully explored.
Micromonas species are part of the 'green' lineage (Chlorophyta) of organisms, a monophyletic group including land plants. Consequently, their physiological response to nutritional stressors, like $\mathrm{P}$ deficiency, may share more traits with land plants than with other algal groups (e.g. diatoms, haptophytes). Indeed, P deficiency is common in lakes and terrestrial systems (Elser et al. 2007). The physiological response of model chlorophytes such as the land plant Arabidopsis thaliana and the freshwater green alga Chlamydomonas reinhardtii to $\mathrm{P}$ deficiency led to the identification of a phosphate starvation response gene described as psr1 in C. reinhardtii (Wykoff et al. 1999) and as phr1 in plants (Rubio et al. 2001). This gene encodes for a transcription factor (TF), a protein that binds to specific DNA regions to activate or repress transcription of one or more genes. The specific region of DNA to which TFs bind is typically characterized by a short repetitive nucleotide sequence, or motif, found up- or downstream of a given gene or within introns (Barrett et al. 2012, Franco-Zorrilla et al. 2014). In A. thaliana, regulatory motifs for the Phr1 protein were more abundant in known P-responsive genes than in the rest of the genome (Müller et al. 2007), linking Phr1 to the regulation of P-responsive genes. Phr1/Psr1 TFs have not been described, to our knowledge, in marine algae.

Here, we use a combined metabolomic and comparative genomic approach to investigate the impact of $\mathrm{P}$ deficiency on the physiology of $M$. pusilla CCMP1545. Many metabolites are produced by metabolic pathways under genetic regulatory control (e.g. De Carvalho \& Fernandes 2010, Markou \& Nerantzis 2013); thus, our approach complements recent physiological, transcriptional, and proteomic work (Guo et al. 2018, Hoppe et al. 2018) and provides mechanistic insight into the physiological response to $\mathrm{P}$ deficiency and its underlying genetic regulation. We used a targeted metabolomics approach to analyze the suite of intracellular and extracellular molecules produced by $M$. pusilla. Informed by our metabolomics results, we employed a comparative genetics approach to identify (1) a psr1-like gene in $M$. pusilla and other prevalent marine phytoplankton species and (2) a potential DNA-binding site of the Psr1-like protein in putatively P-responsive genes. We found evidence for the expression of psr1-like genes in M. pusilla and other marine algae under P-deficient conditions in cultures and in the field. These results have implications for better understanding the metabolic response of diverse phytoplankton groups to $\mathrm{P}$ deficiency. 


\section{MATERIALS AND METHODS}

\subsection{Culture of Micromonas pusilla CCMP 1545}

All glassware was acid-cleaned and combusted at $450^{\circ} \mathrm{C}$ for at least $4 \mathrm{~h}$. We grew an axenic culture of $M$. pusilla CCMP1545 from the National Center for Marine Algae and Microbiota (Boothbay, ME, USA) in L1-Si media (https://ncma.bigelow.org/algal-mediarecipes) with 0.2- $\mu \mathrm{m}$ filtered (Omnipore filters; Millipore) autoclaved seawater from Vineyard Sound (MA, USA). We maintained the cultures at $22^{\circ} \mathrm{C}$ under a 12:12 h light:dark regime (84 $\mu \mathrm{mol}$ photons $\mathrm{m}^{-2} \mathrm{~s}^{-1}$ ). After acclimation to these culture conditions, we split the culture into 2 parallel cultures of L1-Si media amended with (a) $36 \mu \mathrm{M}$ phosphate (P replete) or (b) $0.4 \mu \mathrm{M}$ phosphate (P deficient). We maintained these parallel cultures for 3 generations prior to this experiment. At the start of the experiment, we inoculated 9 flasks of each media type with exponential-phase cells to achieve approximately 300000 cells in each flask in $320 \mathrm{ml}$ total volume $(\mathrm{n}=3$ per treatment for each time point). We also created 3 cell-free control flasks for each treatment. We grew cultures for $2 \mathrm{wk}$ and sampled approximately $1 \mathrm{~h}$ into the light cycle at experiment initiation ( $\mathrm{T}_{0}$; Day 0 ), in late exponential growth phase ( $T_{1}$; Day 4 P deficient; Day 6 P replete), and in stationary phase $\left(\mathrm{T}_{2}\right.$; Day $5 \mathrm{P}$ deficient; Day $13 \mathrm{P}$ replete) (Fig. S1 in Supplement 1 at www.int-res.com/ articles/suppl/a086p029_supp1.pdf).

At each sampling point, we removed $1 \mathrm{ml}$ for cell counts and chlorophyll fluorescence, $30 \mathrm{ml}$ for total organic carbon (Text S1 in Supplement 1), and $20 \mathrm{ml}$ of filtrate (see section 2.2) for nutrients. We monitored cell abundance daily by flow cytometry (Guava, Millipore) and assessed Photosystem II efficiency by measuring the variable and maximum chlorophyll fluorescence $\left(F_{\mathrm{v}} / F_{\mathrm{m}}\right)$ using fluorescence induction and relaxation (Satlantic). We used chlorophyll a (692 nm) and side scatter of M. pusilla cultures to optimize flow cytometry settings. We assessed potential bacterial contamination by viewing DAPI-stained cells at each time point (Text S1).

\subsection{Metabolite extraction and instrument methods}

Metabolite extraction and analytical methods were optimized for semi-polar compounds with a size range of 100-1000 Da, capturing many primary metabolites such as organic acids, vitamins, and nucleosides. We processed cultures for intracellular and extracellular metabolite extraction as described previously (Fiore et al. 2015; Text S1). For intracellular metabolites, we collected cells via filtration on $0.2 \mu \mathrm{m}$ PTFE filters (Omnipore, Millipore) and stored them at $-80^{\circ} \mathrm{C}$. Metabolites were extracted in $500 \mu \mathrm{l}$ of cold 40:40:20 acetonitrile:methanol:water with $0.1 \mathrm{M}$ formic acid. Extracts were reduced to near dryness under vacuum centrifugation and reconstituted in $500 \mu \mathrm{l}$ (extracellular) or $643.5 \mu$ (intracellular) of 95:5 MilliQ water:acetonitrile with deuterated biotin (d2-biotin, final concentration $0.05 \mu \mathrm{g} \mathrm{ml}^{-1}$ ) added as an HPLC injection standard as per Johnson et al. (2017); a $100 \mu \mathrm{l}$ aliquot of the extract was used for targeted metabolomics analysis. We extracted extracellular metabolites using solid phase extraction (SPE) with $1 \mathrm{~g} / 6$ CC PPL cartridges (BondElut, Agilent) as described elsewhere (Dittmar et al. 2008, Longnecker 2015). During the last time point, 3 cultures in the P-replete treatment required multiple filters to process the culture. For these 3 samples, a known volume of culture was passed through each filter. Two filters per sample were used in metabolite extraction, and the extracts were combined prior to metabolomics analysis.

Intracellular and extracellular extracts were analyzed using targeted metabolomics with HPLC (Thermo PAL autosampler and Accela pump) coupled to tandem mass spectrometry (TSQ Vantage, Thermo Fisher Scientific) via a heated electrospray ionization source operated in both positive and negative ion modes (Kido Soule et al. 2015). All 62 standards were quantified using optimized selected reaction monitoring conditions (XCalibur 2.0) in the intracellular samples (Kido Soule et al. 2015), while 23 metabolites with high SPE recovery (Johnson et al. 2017) were quantified in the extracellular samples. Concentrations of metabolites were calculated based on a 5- to 7-point manually curated external calibration curve $\left(0.5-500 \mathrm{ng} \mathrm{ml}^{-1}\right)$. The calibration curve was water-based, and thus we used pooled samples to assess matrix effects. A pooled sample for quality control consisted of 10 parts intracellular extract and 1 part extracellular extract of each experimental sample and was analyzed every 10 injections (Text S1). A laboratory-fortified pooled sample containing a standard mix was also analyzed at the start, middle, and end of the analytical run to assess matrix effects and analytical quality. Chromatographic peaks derived from calibration standards, pooled samples, and experimental samples were manually assessed for quality, and metabolite concentrations were exported to Excel. The limit of detection (LOD) of each compound in our method was determined using Milli-Q water and was estimated for SPE methods with seawater (Johnson et al. 2017). The LOD ranged 
from $\sim 0.4$ to $49 \mathrm{ng} \mathrm{ml}^{-1}$, but most were $<10 \mathrm{ng} \mathrm{ml}^{-1}$ (Johnson et al. 2017). To facilitate comparison with other studies, we also report cell-normalized ratios of the P-deficient to P-replete metabolite concentrations. All metabolomics data are available in MetaboLights (www.ebi.ac.uk/metabolights/) under accession number MTBLS295, and cell- and volume-normalized metabolite concentrations can be found in Dataset S1 in Supplement 2 at www.int-res.com/articles/suppl/ a086p029_supp2.xlsx.

While both intracellular and extracellular metabolites were quantified in our experiment, the results and discussion will focus primarily on changes in the intracellular metabolite profile in response to P deficiency, highlighting compounds that were most relevant to the discovery of the psr1-like gene. We further focus the intracellular metabolite comparison on the exponential growth phase $\left(\mathrm{T}_{1}\right)$ to capture clear changes in metabolite profiles between treatments and facilitate comparison to previous studies (Whitney \& Lomas 2016, Bachy et al. 2018). R statistical software (v3.0.2; R Core Team) was used for statistical analyses. We used a Welch's 2-sample $t$-test, followed by Benjamini-Hochberg false discovery rate correction (Benjamini \& Hochberg 1995), to compare the cell-normalized concentrations of specific metabolites (log transformed to achieve a normal distribution) between P-deficient and P-replete treatments.

\subsection{Identification of the putative phosphate starvation response (psr1) gene and putative DNA-binding motifs}

We used the psr1 gene sequence from Chlamydomonas reinhardtii (Wykoff et al. 1999, NCBI XM_ 001700501.1) to query the genome of $M$. pusilla using the BLAST option through Integrated Microbial Genomes (IMG, blastx, e-value: 1e-5). Conserved domains within putative sequences were characterized using an NCBI conserved domain search (MarchlerBauer \& Bryant 2004). We then used the putative $M$. pusilla psr1-derived amino acid sequence to query several databases using BLAST (tblastn, e-value: 1e-5), including: a re-assembly of the Marine Microbial Eukaryote Transcriptional Sequencing Project (MMETSP) (Johnson et al. 2019), NCBI non-redundant protein sequences (nr), the NCBI metagenome proteins (env $\mathrm{nr}$ ), the Tara Oceans eukaryotic unigenes (Carradec et al. 2018), and several eukaryotic algal genomes from IMG (Text S1). For the Tara Oceans data, we selected only the psr1-like unigenes from the metatranscriptome assemblies that were assigned the taxo- nomic classification of Micromonas. Within each sample, we normalized the expression of the psr1 unigenes to the total abundance of all unigenes taxonomically classified as Micromonas (Text S1). Visualization of the Tara Oceans queries were performed using the 'basemap' library in Python and custom Python scripts (Text S1). For several genes of interest, we compared the distribution of gene expression to the expression of Micromonas psr1-like genes in the Tara Oceans metatranscriptome using a Kolmogorov-Smirnov test. We tested a relationship between phosphate concentration and normalized expression of Micromonas psr1-like genes from Tara Oceans metatranscriptomes by comparing the abundance of Micromonas psr1like transcripts above and below the mean concentration of phosphate using Student's $t$-test.

We manually checked significant matches for all nucleotide sequences that were collected from database-based (NCBI, IMG, MMETSP, Tara Oceans) BLAST searches using the psr1-like gene from $M$. pusilla. First, we used conserved domain analysis (Marchler-Bauer \& Bryant 2004) to assess domain similarity to psr1, and second, we used the sequences with both characteristic domains of the $C$. reinhardtii psr1-derived amino acid sequence in a multiple sequence alignment to the $C$. reinhardtii psr1-derived amino acid sequence. Sequences that did not align with the myb coiled-coil domain of $C$. reinhardtii and $M$. pusilla were removed from further consideration. Sequence alignment and phylogenetic analysis was performed in MEGA 7 (Kumar et al. 2016) using MUSCLE (Edgar 2004) with default settings in MEGA. Phylogenetic reconstruction of the psr1 gene was performed in MEGA using neighbor-joining, maximum parsimony, and maximum likelihood algorithms and bootstrap replication 500 times. The tree topology for the different phylogenetic reconstructions was similar for nodes with high bootstrap values (>50\%, data not shown). We present the maximum likelihood tree because this algorithm (Jones-TaylorThornton model, default maximum likelihood settings in MEGA) uses the most information in its alignments and typically has high accuracy (Felsenstein 1981, Kishino et al. 1990). A FASTA file containing the sequences used in the phylogenetic analysis as well as the alignment exported from MEGA (FASTA format) are available online at https://osf.io/92tg4/.

Following the identification of putative psr1-like genes in $M$. pusilla, we searched genes of $M$. pusilla CCMP1545 for a regulatory binding element, or motif, where the Psr1-like protein would bind to activate or repress gene transcription. The metabolomic results (see Fig. 1) were used to inform our selection 
of genes to be surveyed with the motif analysis. In the survey process, we first searched genes within a now-archived gene model of $M$. pusilla CCMP1545 (FrozenGeneCatalog_20080206 (ver_1), Table S1 in Supplement 1), then we revised our search for binding elements within the updated gene models from Bachy et al. (2018) (M. pusilla CCMP1545/MBARI_ models(ver_1), Table S1 in Supplement 1). Each $M$. pusilla gene sequence used in the motif analysis consisted of concatenated 500 nucleotides upstream and downstream of the gene as well as untranslated regions and intronic regions, thereby excluding the coding regions. We analyzed gene sequences of interest for a significant conserved motif, using the motif-based sequence analysis tools Multiple Em for Motif Elicitation (MEME Suite) 4.10.1 (Bailey et al. 2009). The genes of interest (Table 1) were selected based on metabolites that were, on average, elevated or depressed in concentration in the P-deficient cultures, although they were not statistically different between treatments (i.e. malate, several amino acids, vitamins, see Fig. 1). These included genes related to central carbon metabolism, lipid metabolism, vitamin metabolism (pantothenate, folate), and nucleotide metabolism (aspartate). We also included genes within the carbohydrate and lipid metabolic pathways based on published work (Hockin et al. 2012, Goncalves et al. 2013) as well as the gene for proline oxidase (POX), a key enzyme up-regulated in E. huxleyi in response to N- and P-deficiency (Rokitta et al. 2014, 2016).

After initial motif discovery, we optimized our putative motif by varying motif width and number within genes of interest and within genes that were not expected to be regulated under $\mathrm{P}$ deficiency, including asparagine synthetase (Joint Genome Institute [JGI] gene ID: 9681548) and acetyltransferase-like/flavin adenine dinucleotide-linked oxidase [9687568]). We attempted to identify motifs using the motif comparison tool (Tomtom; Gupta et al. 2007) against the A. thaliana database (Protein Binding Microarray [PBM] motifs, Franco-Zorrilla et al. 2014), and significant matches were used to guide the optimization of motif discovery settings and inclusive sequences. Using the selected sequences and optimized parameters, we searched for motifs that are overrepresented in the query sequences ('positive') relative to a set of background sequences ('negative'), the latter defined using the genome scaffolds. We used positive and negative sequences to create a position specific prior distribution file (Bailey et al. 2009), for further discriminative motif discovery within $M$. pusilla gene sequences of interest (parameters: $\operatorname{minw}=5$, maxw $=10$, nmotifs $=$ $3, \bmod =$ oops [one occurrence per sequence]).
Two recent publications provided transcriptomes for testing our hypotheses regarding expected differentially expressed (DE) genes in P-deficient and Preplete conditions. Whitney \& Lomas (2016) published transcriptomes of $M$. commoda (formerly M. pusilla) RCC299, while Bachy et al. (2018) published transcriptomes of M. pusilla CCMP1545, under P-deficient or P-limiting (respectively) and P-replete conditions. Genes discussed here as DE were defined by each study (Whitney \& Lomas 2016, Bachy et al. 2018). We first compared our hypothesis for genes that would be differentially regulated in $M$. pusilla to observations in Bachy et al. (2018). We also used the information from Bachy et al. (2018) to inform our targeted search for a significant conserved motif in genes that may be regulated by the Psr1-like TF. We note that each Micromonas experiment used slightly different growth conditions and were sampled at different stages of growth. Thus, we focus our comparisons on work in the same strain (M. pusilla CCMP1545, Bachy et al. 2018), with brief complementary discussions about $M$. commoda (expanded results in Text S2 in Supplement 1). Bachy et al. (2018) used semi-continuous cultures grown under continuous light and with a moderately higher concentration of $\mathrm{P}(2.5 \mu \mathrm{M})$ than in our experiment $(0.4 \mu \mathrm{M})$. Despite these differences, the transcriptional data of $M$. pusilla under P limitation provided a preliminary test of our hypotheses derived from the metabolomics analyses with $M$. pusilla CCMP1545 and in silico genomic analysis. We further reasoned that a signal observed across species and experiments would likely represent a conserved and biologically meaningful response to P deficiency.

\section{RESULTS}

\subsection{Metabolic response of Micromonas pusilla CCMP1545 to P deficiency}

Quality control analysis of the d2-biotin injection standard described above indicated low ionization variability across the pooled samples (coefficient of variation $[\mathrm{CV}]=8.5 \%$ ) and the intracellular samples $(\mathrm{CV}=20 \%)$, but higher variability across extracellular samples $(\mathrm{CV}=65 \%)$. This is likely a result of the dissolved organic carbon background from seawater in the extracellular extracts (but absent in the intracellular) coeluting with d2-biotin, causing matrix interference. Previous work using spent media from M. pusilla observed no significant matrix effect on the instrument response factor for most of the metabolites assessed by the same targeted metabolomics 
Table 1. Enzymes that contain a significant motif in the gene sequences of Micromonas pusilla CCMP1545 (MBARI_models (ver 1)) and contain a putative myb-like DNA-binding domain. EC number: Enzyme Commission number or pfam identifier; pathway: main pathway(s) in which the enzyme is involved. Fold change and $q$-values of the transcript in P-deficient relative to P-replete conditions are data from Bachy et al. (2018) and are given with the corresponding Joint Genome Institute (JGI) gene ID. CoA: coenzyme A; TCA: tricarboxylic acid; ETC: electron transport chain

\begin{tabular}{|c|c|c|c|c|c|}
\hline Enzyme & $\begin{array}{c}\text { EC } \\
\text { number }\end{array}$ & Pathway & $\begin{array}{l}\text { Log2 fold } \\
\text { change }\end{array}$ & $q$-value & $\begin{array}{c}\text { JGI } \\
\text { gene ID }\end{array}$ \\
\hline $\begin{array}{l}\text { Glyceraldehyde-3-phosphate } \\
\text { dehydrogenase }\end{array}$ & 1.2.1.12 & Calvin cycle/glycolysis & 2.73 & 0.000037 & 4267 \\
\hline $\begin{array}{l}\text { Glyceraldehyde-3-phosphate } \\
\text { dehydrogenase }\end{array}$ & 1.2.1.12 & Calvin cycle/glycolysis & -0.644 & 0.000037 & 700 \\
\hline Phosphoglycerate kinase & 2.7 .2 .3 & Glycolysis & -0.699 & 0.000037 & 7661 \\
\hline Pyruvate kinase & 2.7 .1 .40 & Glycolysis & -1.32 & 0.000037 & 9370 \\
\hline Pyruvate dehydrogenase & 1.2 .4 .1 & TCA cycle/glycolysis & 0.756 & 0.000037 & 3535 \\
\hline Citrate synthase & 2.3.3.1 & TCA cycle & 1.68 & 0.000037 & 1278 \\
\hline Citrate synthase & 2.3.3.1 & TCA cycle & 1.10 & 0.000037 & 3714 \\
\hline Aconitase & 4.2 .1 .3 & TCA cycle & 1.00 & 0.000037 & 2901 \\
\hline Succinate dehydrogenase & 1.3 .5 .1 & TCA cycle and ETC & 1.16 & 0.001515 & 2182 \\
\hline Fumarase & 4.2 .1 .2 & TCA cycle & 1.68 & 0.000037 & 600 \\
\hline Fumarase & 4.2.1.2 & TCA cycle & 1.73 & 0.000068 & 6662 \\
\hline Copper amine oxidase & $1.4 .3 .21-22$ & Arginine and proline metabolism & 2.87 & 0.000037 & 5863 \\
\hline Aspartate transcarbamylase & 2.1.3.2 & Pyrimidine biosynthesis & -0.609 & 0.000037 & 3615 \\
\hline Nucleoside phosphatase & 3.6 .1 .3 & Pyrimidine metabolism & 0.711 & 0.000037 & 3322 \\
\hline Pantoate-beta-alanine ligase & 6.3 .2 .1 & Pantothenate and CoA biosynthesis & -0.616 & 0.000037 & 5304 \\
\hline Lysophospholipase & 3.1 .1 .5 & Glycerophospholipid metabolism & 1.65 & 0.000037 & 3652 \\
\hline Acyl-CoA synthetase & 6.2 .1 .3 & Fatty acid metabolism & 3.20 & 0.000037 & 1751 \\
\hline $\begin{array}{l}\text { Long-chain acyl-CoA } \\
\text { synthetases }\end{array}$ & 6.2 .1 .3 & Fatty acid metabolism & 0.736 & 0.000037 & 416 \\
\hline $\mathrm{Na}+/ \mathrm{PO}_{4}$ transporter & PF02690 & Inorganic nutrient transport & 4.54 & 0.000037 & 5963 \\
\hline
\end{tabular}

method that we used here (Johnson et al. 2017). In this experiment, we quantified 21 metabolites from that study (Fig. S2 in Supplement 1) and of these, 8 were shown to exhibit ionization suppression in the M. pusilla extracellular extracts (Johnson et al. 2017). Thus, we have used caution in the interpretation of the extracellular metabolite comparisons between treatments (Fig. S2).

Metabolite concentrations in the extracellular fraction (Fig. S2) were generally lower under P deficiency relative to the P-replete cultures, although these differences were not statistically significant between treatments ( $t$-test, $\mathrm{p}>0.05$ ). The extracellular metabolite concentrations were standardized to the number of cells in each sample and represent cumulative changes in the media over time. We note that some core metabolites may be present below the limit of detection of our method (Johnson et al. 2017).

In contrast, there was a mixed response in metabolite concentrations in the intracellular fraction. Several of the intracellular metabolites, standardized to cell number, were abundant under $\mathrm{P}$ deficiency during exponential growth $\left(\mathrm{T}_{1}\right)$, including nucleobases (xanthine, adenine), the nucleoside inosine, amino acids (glutamine, aspartate), the amino acid derivatives $N$ - acetylglutamate and tryptamine, the dicarboxylic acid malate, and vitamins (thiamin, pantothenate, folate) (Fig. 1). Due to high variability in our treatments, we did not observe statistically significant results after $\mathrm{p}$-value correction ( $t$-test, $\mathrm{p}>0.05)$. However, we observed decreases in the intracellular ratios of purine nucleosides (guanosine, xanthosine) to their nucleobases (guanine, xanthine) under $\mathrm{P}$ deficiency at $\mathrm{T}_{1}$ (Fig. 2). The decrease in the xanthosine:xanthine ratio was statistically significant ( $t$-test, $p=0.03$ ). The change in the guanosine:guanine ratio could not be statistically tested, as guanine was below detection in 2 of 3 P-replete cultures (Fig. 2). The di- and tricarboxylic acids malate, succinate, and citrate exhibited divergent responses, although their abundances were not significantly different between treatments (Fig. 1). Malate was more abundant in P-deficient cells, while succinate concentrations were similar between treatments and replicates; citrate was detected in only 1 of 3 replicates in each treatment (Fig. 1). We noted similarly varied responses to $\mathrm{P}$ deficiency in the purine nucleosides xanthosine and inosine, with invariant xanthosine abundances but higher average concentrations and variability of inosine (Figs. $1 \& 2$ ). 


\subsection{Characterization of the phosphate starvation response (psr1) gene in marine algae}

We found 4 statistically significant sequences (Table 2) in response to querying the now-archived M. pusilla CCMP1545 genome with the psr1 gene from Chlamydomonas reinhardtii (strain cc125, NCBI accession AF174532). One of these sequences (JGI ID 61323) had the 2 characteristic myb domains of the

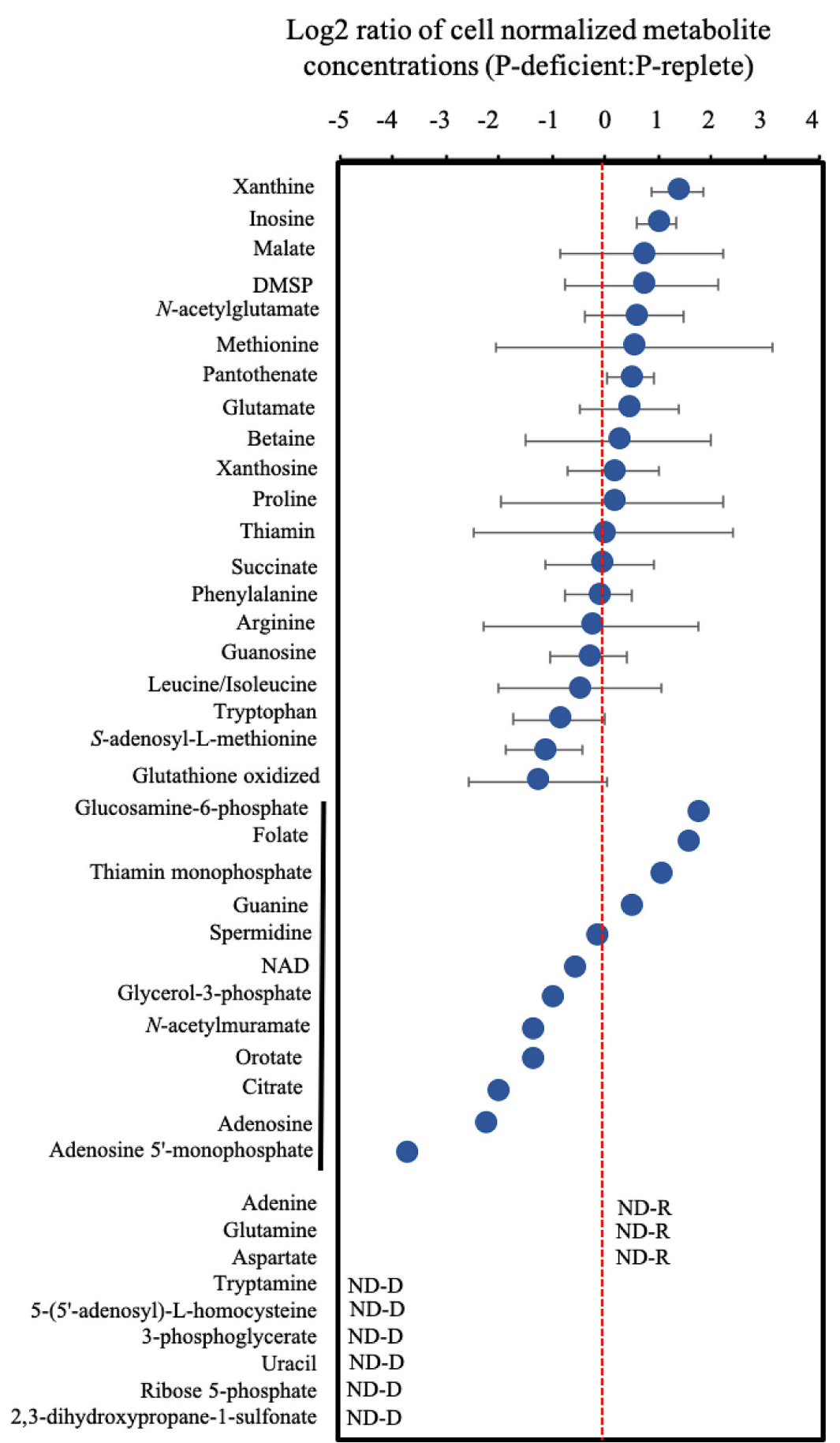

psr1 gene in C. reinhardtii (myb-like DNA-binding domain, SHAQKYF class [TIGR01557] and myb predicted coiled-coil type transfactor, LHEQLE motif [pfam PF14379]) (Fig. 3A). However, the helixturn-helix dimerization domain and glutamine-rich regions that are characteristic of TF activation domains, as well as the putative metal-binding domain originally described for $p s r 1$ in C. reinhardtii (Wykoff et al. 1999) were not detected in the putative $M$. pusilla psr1 gene. Thus, we refer to the gene in $M$. pusilla as a psr1-like gene (Fig. 3; Fig. S3 in Supplement 1). Conserved domain analysis (Marchler-Bauer \& Bryant 2004) revealed 2 other domains in the putative psr1like-derived amino acid sequence from M. pusilla: (1) PLN03162 super family (NCBI cl26028), a provisional golden2 like $\mathrm{TF}$, and (2) a DUF390 super family domain (NCBI cl25642), comprising proteins of unknown function (Fig. S3). A comparison of homology of the psr1-like gene between $M$. pusilla, M. commoda, C. reinhardtii, and Arabidopsis thaliana is provided in Table S2 in Supplement 1.

We identified putative psr1-like genes or transcripts in cultured isolates as well as in field datasets (see Figs. 3b, 4, \& 5; Table S3 in Supplement 1),

Fig. 1. Log 2 of the average ratio of the Pdeficient to P-replete intracellular metabolite concentrations during exponential growth $\left(\mathrm{T}_{1}\right)$ in Micromonas pusilla CCMP1545. Concentrations were normalized to cell number in each treatment and means ( \pm propagated uncertainty) are shown ( $\mathrm{N}=3$ unless otherwise noted). Red dashed line indicates a ratio of 0 , where the concentrations of the metabolites are the same between treatments. Metabolites are listed in order of descending value of the ratio within 3 groups: those that were detected in enough replicates for uncertainty to be calculated, those where only 1 ratio could be calculated (black line), and those that were not detected (ND) in any of the 3 replicates for one treatment $(\mathrm{R}=\mathrm{P}$ replete, $\mathrm{D}=\mathrm{P}$ deficient). Only 1 replicate in the P-deficient treatment contained a non-0 concentration for $N$-acetylmuramic acid; thus, while there was a significant difference in concentration for this metabolite, only 1 ratio could be calculated. DMSP: dimethylsulfoniopropionate; NAD: nicotinamide adenine dinucleotide 


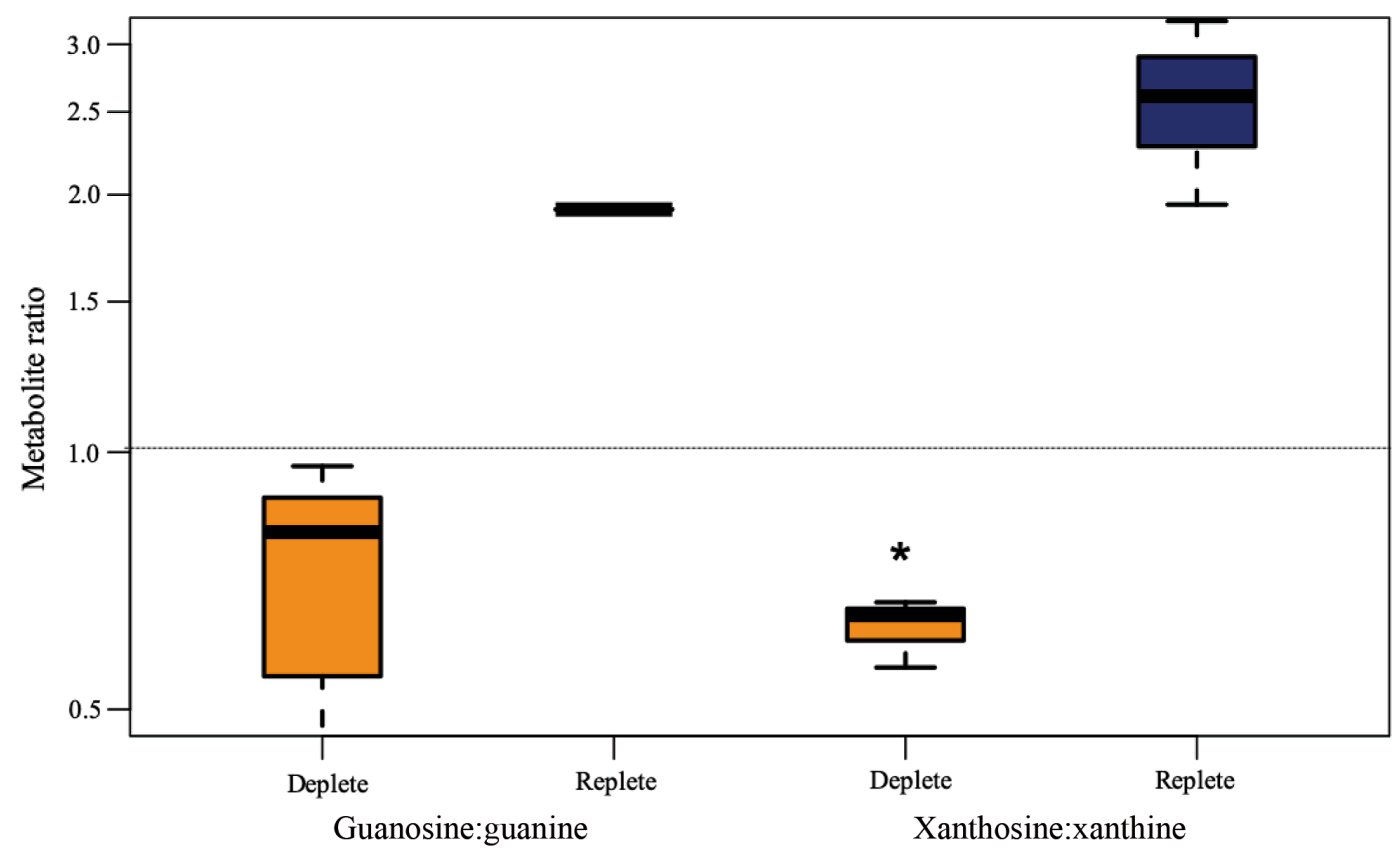

Fig. 2. Intracellular ratios of 2 purine nucleosides to their nucleobases for Micromonas pusilla CCMP1545 in P-deficient and P-replete treatments during exponential growth $\left(T_{1}\right)$. The box extends from the first quartile to the third quartile with a thick line at the median, and the whiskers represent the minimum and maximum values. Xanthosine and xanthine, and guanosine and guanine were quantified in the targeted metabolomics method and normalized to cell abundance. The average ratio and high and low values are shown based on 3 replicate cultures. The asterisk marks a significant difference between the treatments $(t$-test, $\mathrm{p}=0.03)$. The dashed line represents a metabolite ratio between treatments of 1 . Two samples in the P-replete treatment had guanine levels below the limit of detection and the one ratio is shown as a line

Table 2. Gene matches to psr1 in Micromonas pusilla CCMP1545 as originally discovered in the Joint Genome Institute (JGI) Integrated Microbial Genomes (IMG) database and the corresponding gene in the updated gene model for CCMP1545 (MBARI_models (ver 1)). The M. pusilla CCMP 1545 database in IMG contained 10660 sequences and 4795637 letters. The top-scoring gene was considered to be a psr1-like gene and the JGI gene ID is shown in parentheses. The corresponding psr1-like gene in the updated gene model is shown for reference, and includes the JGI gene ID, the gene description, and the Evalue from the BLAST search (see Section 2) using the top scoring gene (61323) from CCMP1545

\begin{tabular}{|llcc|}
\hline IMG gene ID & Locus tag & Bit score & E-value \\
\hline 2615011133 & MicpuC2.est_orfs.1_306_4269596:1 & 90.5 & $2 \times 10^{-19}$ \\
(JGI 61323) & & & \\
2615008475 & MicpuC2.EuGene.0000130210 & 74.3 & $4 \times 10^{-14}$ \\
2615010887 & MicpuC2.est_orfs.10_1861_4270447:1 & 70.5 & $3 \times 10^{-13}$ \\
2615007841 & MicpuC2.EuGene.0000090108 & 67.8 & $4 \times 10^{-12}$ \\
JGI ID 360 & Myb domain-containing protein & & $8 \times 10^{-156}$ \\
\hline
\end{tabular}

representing diverse taxa such as Dinophyta (e.g. the symbiotic clade C Symbiodinium sp.) and Haptophyta (e.g. the coccolithophore Emiliania huxleyi). In our phylogenetic analysis (Fig. 4), sequences generally clustered by taxonomic group with multiple clades of Chlorophyta across the tree (Fig. 4). However, the clades with other groups such as haptophytes (e.g.
Chrysochromulina rotalis and E. huxleyi) are not well resolved (Fig. 4; Fig. S4 in Supplement 1). There were also relatively few non Chlorophytaderived sequences that could be included in the alignment due to low quality (i.e. short sequence length).

Transcripts from MMETSP and Tara Oceans, as well as high-identity hits to sequences from the Global Ocean Sampling expedition (GOS, Rusch et al. 2007), were generally short sequences and often contained 1 of the 2 characteristic domains for $p s r 1$-derived amino acid sequences (Text S2). Field-derived transcripts of the psr1-like gene were geographically dispersed in the Tara Oceans dataset with the highest relative expression in surface samples and in the North Atlantic Ocean and Mediterranean Sea (Fig. 5A). The majority of these transcripts from the Tara Oceans dataset occurred in samples with low phosphate concentrations $(\leq 0.5 \mu \mathrm{M})$, but there was no significant relationship between psr1-like gene expression and phosphate concentration $(t$-test, $\mathrm{p}<0.05$; 
MTRMSGIEDDSFSYSFLLISGQLRDPTRLTPRPQTSSAPLGAGGGAAAASGMNTSGTFWPSDLDLDLESSDFL DSLLLGGEMMTTQQQHHHAGDERLGHGPVPLGARAAGEDHDPRVVLAERDAGDGLLPRALADERREPVAR AVVASNNNKQRLRWTPELHKMFVDAVKRLGGLDLATPKGIMQLMDVEGMSIQHVKSHLQKYRLQDSGGG ASEFRVSPDASASGKRPRSEEDDAGGNGKDGNNSAGKTRRRPSAAERSAARLRAAEEKAREERDAARSMAA AAAAAAAAAVERQNVELALLTGDAAGARDALEMSSHHAASHVGGAYDDVLDLVAGPGESGDGDGAWGD VLHDHAIVGAAGDDVGLVDDVGSDPEAAAAMLKQLELQKKLHEHLMSQRRLQQQVEAHGVYLETILDQQK RRRGVE

B)

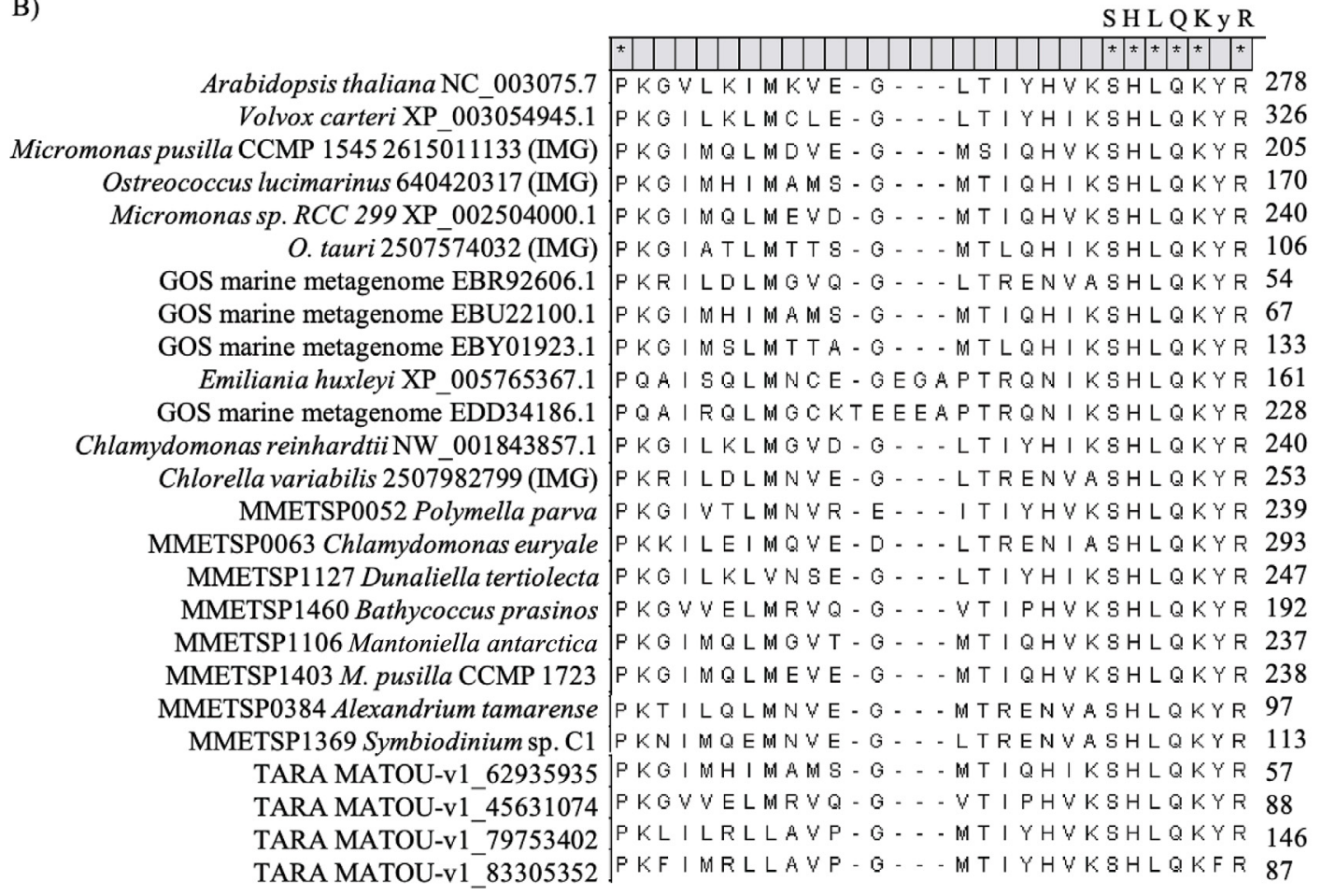

L H E Q L

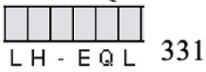

\begin{tabular}{ll}
$\mathrm{LH}-\mathrm{EQL}$ & 331 \\
\hline $\mathrm{H}-\mathrm{EOL}$ & 457
\end{tabular}

LH - EHL 396

LH. DQL 320

LH.AQL 416

LH.DQL 241

PDG GM M 201 *

. . . . 84 *

LHSMDA 223 *

LE - . - 212*

$L H-E Q Q 327$ *

LH - E Q L 403

. . . . 334 *

TANATL 425 *

GG - . - 361*

FQGAGG 335 *

LH - EQL 452

SA-AR। 332 *

LH - EQL 413

M G . . . 176 *

. . . . 110 *

‥ - 74 *

LH - EQL 348

L Q - EQ L 243

$L R \cdot E Q L 158$ *

Fig. 3. Predicted amino acid sequences of the psr1-like gene in Micromonas pusilla CCMP1545 and a subset of other organisms. The amino acid sequence (with IMG gene ID) is shown as described in the Joint Genome Institute Integrated Microbial Genomes M. pusilla CCMP1545 database. (A) The myb-like DNA-binding and myb coiled-coil domains are highlighted in blue. (B) Predicted amino acid sequence alignment of psr1 and psr1-like genes in the region of the myb-like DNA-binding domain (SHAQKYF class) and LHEQL coiled-coil domain. Note that not all sequences contain the LHEQL domain as characterized by CD-Search (Marchler-Bauer \& Bryant 2004); these are marked with an asterisk on the right side of the alignment. Asterisks at the top of each column indicate $100 \%$ conserved residues across species surveyed. Numbers at the end of each row indicate position of the last shown residue in the amino acid sequence. Accession (GOS, MMETSP, NCBI, Tara) or gene identification (IMG) numbers are listed for each sequence

Fig. 5B). We observed a significant positive relationship (Kolmogorov-Smirnov, p < 0.05) between the presence of Micromonas psr1-like genes and the relative expression of fumarase, a tricarboxylic acid (TCA) cycle gene in Micromonas, which was up-regulated in cultures of M. pusilla (Bachy et al. 2018) and M. commoda (Whitney \& Lomas 2016) under P deficiency (Fig. 5C; Text S3 in Supplement 1). We did not find genes with significant homology to the $M$. pusilla putative psr1-like gene, in genomes or transcriptomes, within diatoms, the Heterokonta superphylum, or the cryptophytes.

\subsection{Putative regulatory element in M. pusilla genes of interest}

MEME analysis yielded a significant conserved motif in M. pusilla genes involved in central carbon metabolism, lipid metabolism, and nucleotide metabolism (Fig. S5 in Supplement 1). The genes of interest for MEME analysis (Table 1) were selected based on our metabolomics results and informed by the literature (see Section 2.3). The significant conserved motif is similar in nucleotide sequence to several TFbinding sites in A. thaliana (Table S4 and Fig. S6 in 


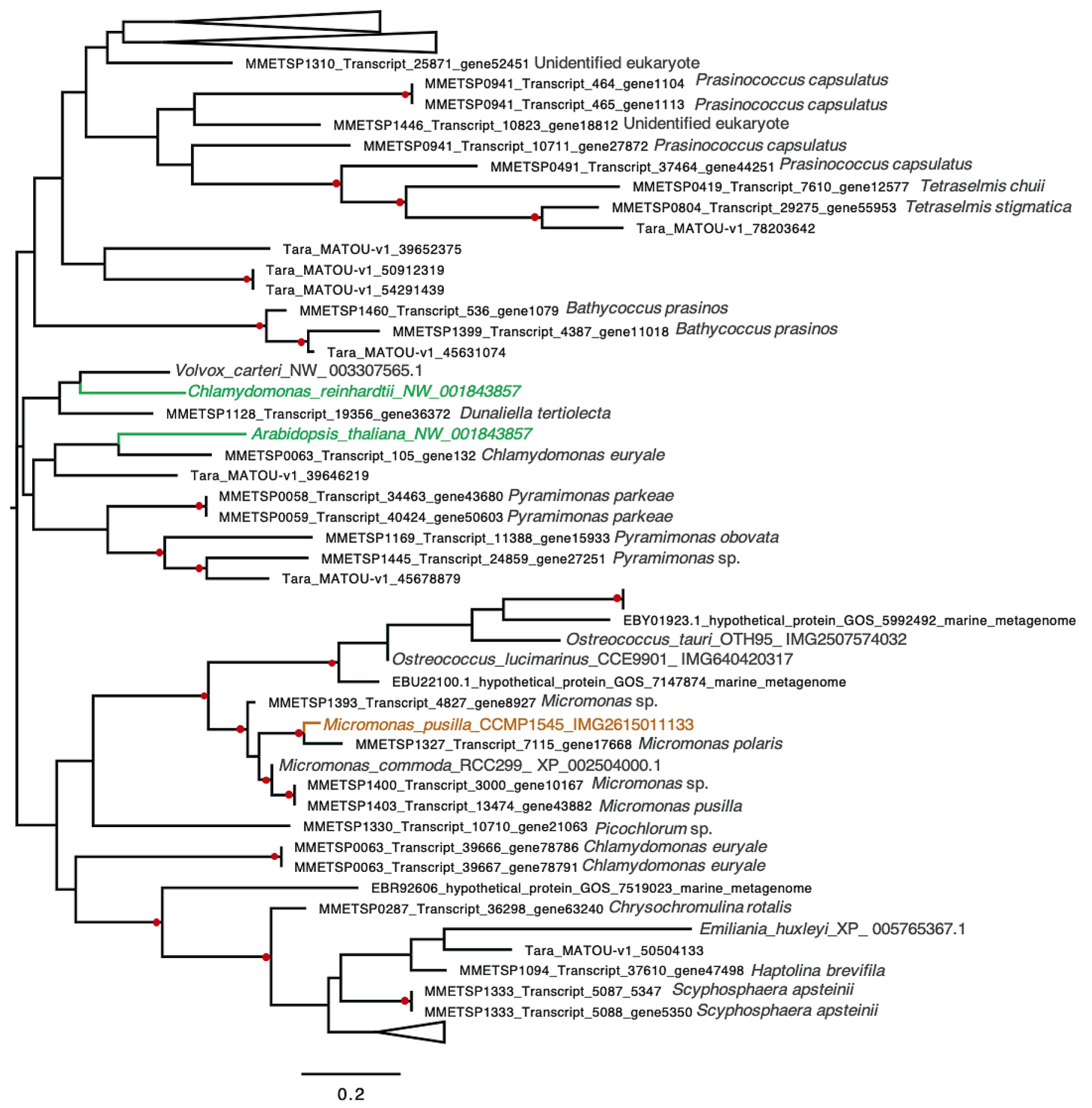

Fig. 4. Occurrence and phylogenetic relationship of psr1-like genes in marine phytoplankton. All sequences that included the conserved SHLQKYR and LHEQL domains (see Fig. 3) were included in the alignment (see Section 2 for details), with the exception of 2 global ocean survey (GOS) sequences and the Emiliania huxleyi sequence. These sequences were short sequences and only contained the SHLQKYR domain, but were included for context. Several branches are collapsed for clarity and the full tree is shown in Fig. S4. Maximum likelihood method is based on the JTT matrix-based model (Jones et al. 1992). The tree with the highest log likelihood (-4344.61) is shown and is based on the derived amino acid sequences for psr1 and psr1-like genes from eukaryotic phytoplankton. Bootstrap support for branches that are $\geq 50 \%$ are marked with a red circle. The tree is unrooted and drawn to scale, with branch lengths measured in number of substitutions per site. The analysis involved 80 amino acid sequences. All positions containing gaps and missing data were eliminated. The final dataset included a total of 66 positions. Evolutionary analyses were conducted in MEGA7 (Kumar et al. 2016). Micromonas pusilla CCMP1545 used in this study is colored brown, and genes that have been functionally characterized are colored green (Rubio et al. 2001, Moseley et al. 2006, Bajhaiya et al. 2017). 
A) Normalized psr1-like abundance (proportion of Micromonas transcripts)
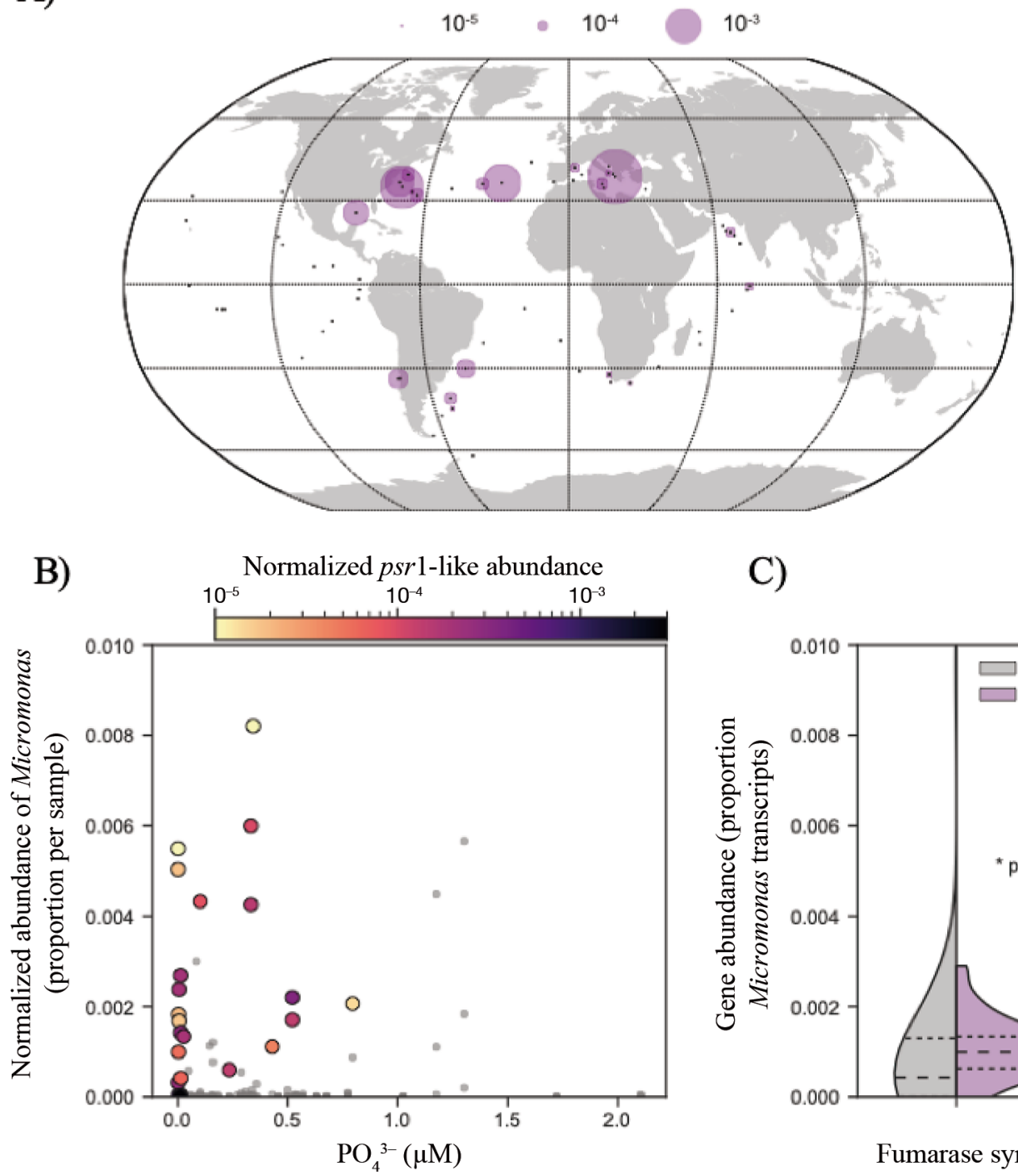

C)

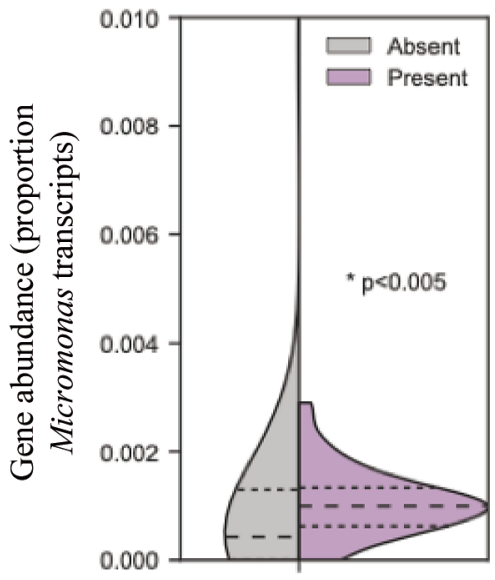

Fumarase synthase

Fig. 5. Geographic distribution of Micromonas psr1-like genes and relationship with phosphate concentration in the Tara Oceans eukaryotic gene atlas (Carradec et al. 2018). (A) Geographic distribution and relative abundance of Micromonas psr1-like genes in surface samples. Black dots represent stations where Micromonas was detected; the size of purple circles represents the relative abundance of the psr1-like gene, where the fragments per kilobase million (FPKM) of the psr1-like gene was normalized to the total FPKM of all Micromonas-associated transcripts. (B) Relative abundance of Micromonas plotted against phosphate concentrations for surface stations where Micromonas was detected. Grey circles indicate samples where psr1-like genes were not detected, while samples where psr1-like genes were detected are colored based on the relative expression of the Micromonas psr1-like gene, again normalized to total Micromonas transcript abundance. No significant relationship was observed between the phosphate concentration and abundance of Micromonas psr1-like transcripts ( $t$-test, p > 0.05). (C) Distribution of the expression of Micromonas fumarase synthase genes as a function of presence or absence of the psr1-like gene. The larger dashed line represents the distribution mean and the smaller dashed lines represent the 25 and $75 \%$ quartiles. Significant difference in the

distribution of expression between the presence and absence was assessed with a Kolmogorov-Smirnov test $(p<0.005)$

Supplement 1, Franco-Zorrilla et al. 2014), including myb-like TF-binding motifs. The presence of a myblike TF-binding motif is notable as the Psr1 protein contains a myb-like DNA-binding domain (Wykoff et al. 1999). Several iterations of this analysis using genes not tied to the metabolites of interest yielded either no significant motif or motif sequences lacking similarity to myb-like domains. We observed a similar significant conserved motif in author-defined DE transcripts (Bachy et al. 2018) in M. pusilla CCMP1545 under P deficiency (Table 1; Fig. S5). While the actual nucleotide sequence recognized by a TF must be experimentally determined (FrancoZorrilla et al. 2014), the presence of a significant 
conserved motif in each model, and only in the expected genes, indicates that this motif is likely biologically significant in $M$. pusilla. Specifically, this motif, with similarity to myb-like domains in $A$. thaliana, may represent a regulatory element where the Psr1-like protein binds.

\subsection{Comparison of metabolomics-based predictions of P-responsive genes in M. pusilla CCMP1545 to transcriptomics analysis}

We compared the author-defined DE transcripts in M. pusilla CCMP1545 to gene predictions based on our metabolomics data. As Bachy et al. (2018) used a more recent gene model than our original analysis, we confirmed the identity of the psr1-like gene in the updated model (CCMP1545/MBARI models(ver_1), Table 2). We then analyzed a similar set of genes for a regulatory element where the Psr1-like TF might bind (Fig. S5), including genes that were DE in the P-deficient transcriptome relative to the replete transcriptome (Table S1 from Bachy et al. 2018). Under P deficiency, the psr1-like gene (JGI gene ID 360) was highly up-regulated (log-2 fold-change $=4.3, q=0.00037$ ), and nearly all of the predicted P-responsive genes were DE (Table 1). For example, $M$. pusilla up-regulated many genes in the TCA cycle but not pyruvate carboxylase (gene ID 6984). Genes involved in nucleotide and lipid metabolism (e.g. nucleoside phosphatase, long-chain acyl-coA synthetase) were DE and contained a significant conserved motif with similarity to the myb-like regulatory element in A. thaliana (Table 1, Fig. S5). Lastly, the POXencoding gene described in E. huxleyi (Rokitta et al. 2016) and observed in the archived gene model for $M$. pusilla, was absent, or not similarly annotated in the updated gene model. Instead, a copper amine oxidase (gene ID 5863) contained a significant conserved motif (Table 1, Fig. S5) and was DE under P deficiency (Bachy et al. 2018).

We conducted a similar analysis with published transcriptome data from $M$. commoda (Whitney \& Lomas 2016). The psr1-like gene (JGI gene ID 60184) in $M$. commoda was significantly up-regulated in the P-deficient treatment (defined by Whitney \& Lomas 2016; Table S5 in Supplement 1), and we found a significant conserved motif in 18 DE genes. The significant conserved motif identified in $M$. commoda differed in sequence from that discovered in $M$. pusilla and occurred in a different set of genes (Text S2).

\section{DISCUSSION}

Our combined metabolomics and comparative genomic analyses of the response of Micromonas pusilla CCMP1545 to P deficiency has led to 3 conclusions: (1) there was an observable shift in intracellular metabolite composition, (2) a psr1-like gene is expressed in M. pusilla and other marine phytoplankton, and (3) the genes regulated by the Psr1like protein may differ amongst algal species.

\subsection{M. pusilla exhibits a metabolic shift in response to $\mathbf{P}$ deficiency}

In the extracellular media, some metabolites such as guanosine, pantothenate, 2,3-dihydroxybenzoate, and leucine, and inosine were lower in concentration on average in the extracellular P-deficient treatment, but there were no significant differences between treatments. A reduction in many metabolites involved in central metabolism would be expected, as nutrient-limited microalgae may have lower photosynthetic activity (Loebl et al. 2010, Halsey et al. 2014, Guo et al. 2018) and hence produce less metabolic overflow. The high variability across replicate cultures is likely a factor in the lack of significance in concentration between treatments, and there may be other metabolites not quantified here that are significantly different in concentration between treatments. The lack of significant differences in the quantified extracellular metabolites could also indicate regulation of cellular metabolism that impacts metabolic overflow.

The intracellular metabolites did not differ in concentration between treatments; however, we found examples of functionally related metabolites that behaved differently. For example, in the TCA cycle, malate was variable but higher in concentration on average under $\mathrm{P}$ deficiency, while citrate was lower on average and succinate was relatively unchanged. The opposing responses of these TCA intermediates was notable given that others (e.g. Hockin et al. 2012, Goncalves et al. 2013, Alipanah et al. 2018) have observed a coordinated response to nutrient limitation in transcripts or proteins involved in the TCA cycle. Specifically, the diatom Phaeodactylum tricornutum was observed to down-regulate genes involved in the TCA cycle with simultaneously low concentrations of TCA cycle intermediates under $\mathrm{P}$ deficiency (Alipanah et al. 2018). We also observed additional non-uniform metabolite responses, with concentrations of some vitamins (e.g. pantothenate) and amino acids and derivatives (e.g. malate, $N$ - 
acetylglutamate) higher on average under $\mathrm{P}$ deficiency, while other vitamins and organic acids (e.g. thiamin, $N$-acetylmuramate) were depleted or more variable across treatments (Fig. 1). Metabolites that contain $\mathrm{P}$, such as adenosine $5^{\prime}$-monophosphate, glycerol-3-phosphate, and 3-phosphoglycerate were generally depleted and variable in concentration in the P-deficient treatment (Fig. 1).

Previous work has suggested that organisms placed under nutrient stress are capable of diverting carbon flux between pathways (reviewed by Markou \& Nerantzis 2013). While metabolite concentrations are regulated at multiple levels, including post-transcriptional modification and enzymatic regulation, another means is through transcription of enzyme-encoding genes. If the shunting of carbon is a coordinated response to $\mathrm{P}$ deficiency within $M$. pusilla, then it is possible that genes involved in the synthesis and/or catabolism of these metabolites are co-regulated, and potentially mediated by a specific TF. Motivated by the description of a phosphate starvation response gene (psr1) containing a myb DNA-binding domain by Wykoff et al. (1999), and by the non-uniform metabolite patterns that we observed, we investigated the presence of a psr1 gene and a myb-like motif in genes that could be regulated by the psr1 gene product in M. pusilla. Indeed, we discovered a gene in M. pusilla and other marine algae with significant homology to the TF phosphorus starvation response gene ( $p s r 1$ ) originally described in the freshwater alga Chlamydomonas reinhardtii (Wykoff et al. 1999).

\subsection{Presence and expression of the TF gene psr 1 in marine algae}

In C. reinhardtii and in many plants, a psr1 gene encodes for a TF (Psr1) that coordinates the metabolic response to $\mathrm{P}$ deficiency in these organisms (Wykoff et al. 1999, Moseley et al. 2006, Bajhaiya et al. 2017). We detected a putative psr1-like gene in the $M$. pusilla genome, as well as in the genomes and transcriptomes of major algal lineages (e.g. prasinophytes, dinoflagellates). The $p s r 1$-like genes that we discovered were generally not annotated as psr1, with the exception of Ostreococcus tauri (Derelle et al. 2006). To our knowledge, however, the potential role of this gene in the metabolism of $O$. tauri or other marine algae has not been discussed. No psr1-like gene was identified by our queries of diatom transcriptomes or genomes, suggesting that a phosphate starvation response gene akin to psr1 is present in diverse but phylogenetically constrained phytoplankton groups.
We found significant similarity between the $M$. pusilla psr1-like gene and transcript sequences from the MMETSP and Tara Oceans metatranscriptomic datasets, indicating that this gene is expressed by marine algae in both cultures and in the environment. High-identity hits to sequences in the metagenomic dataset from the GOS (Rusch et al. 2007) and the Tara Oceans metatranscriptomes underscore the prevalence of this gene in the oceans, particularly in regions characterized by chronically low $\mathrm{P}$ concentrations. Short sequences in some MMETSP, GOS, and Tara Oceans sequences did not include enough of the C-terminal end to capture the myb coiled-coil domain. Thus, further investigation is required to confirm whether the identified transcripts are from psr1-like genes. Several GOS sequences, previously described as protein of unknown function, had homology to one of the characteristic Psr1 domains. It is possible that with further characterization, they could be re-annotated as psr1-like genes. The prevalence of psr1-like genes in the field datasets in particular highlights the need to elucidate the role of a psr1-like gene in these organisms.

\subsection{Potential role of Psr1 in the marine algal response to $P$ deficiency}

TFs typically recognize a specific motif in DNA where the protein binds to regulate each gene. If such a motif is observed, one can hypothesize that a gene containing the motif is regulated by the protein. We observed a conserved and enriched motif across genes involved in nucleotide biosynthesis, the TCA cycle and glycolysis, carbon fixation, fatty acid metabolism, and phosphate transport or salvage (Fig. 6). The significant conserved motifs discovered in both M. pusilla and M. commoda had unique sequences and were present in a slightly different set of genes (Text S3). The motif detected in M. pusilla is similar in nucleotide sequence to a myb regulatory element in Arabidopsis thaliana. This similarity is potentially significant, because the $p s r 1 / p h r 1$ genes in C. reinhardtii and A. thaliana contain a myb DNA-binding domain. Thus, we hypothesize that the significant conserved motif detected in the Micromonas species represents binding sites for the Psr1-like TFs.

We observed the significant conserved motif in both Micromonas species only in DE genes under P deficiency (Whitney \& Lomas 2016, Bachy et al. 2018), suggesting that these genes could be co-regulated. In bacteria, for example, genes within the wellcharacterized phosphate (Pho) regulon all contain 


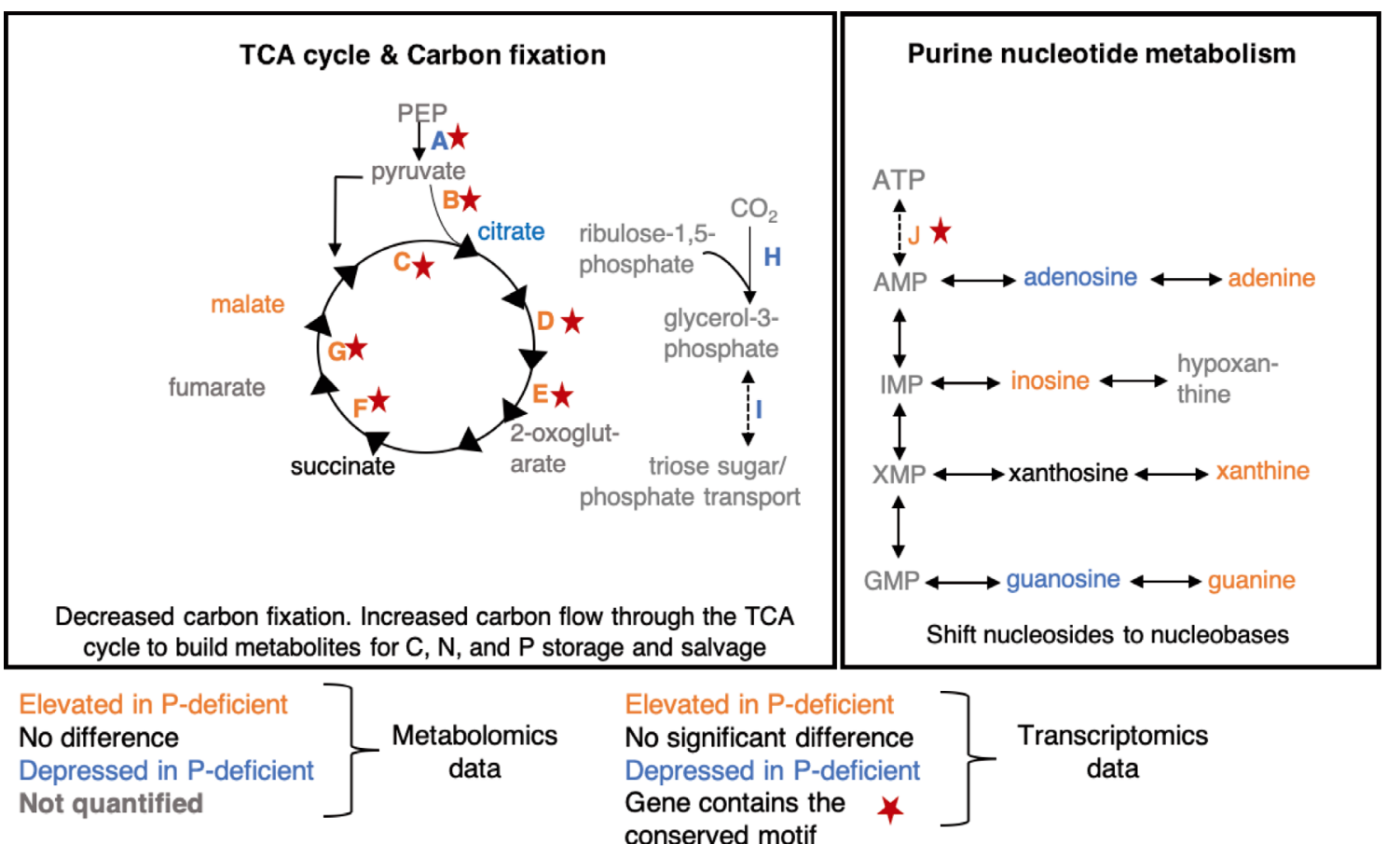

Fig. 6. Conceptual model of Psr1-like transcription factor regulation in Micromonas pusilla CCMP154 in 2 major metabolic pathways under phosphorus deficiency. The figure illustrates the relevant metabolites quantified in the present study and indicates if they are elevated (orange, ratio $>0$; Fig. 1) or depressed (blue, ratio $<0$; Fig. 1) in concentration in the P-deficient cells relative to the P-replete cells. Some metabolite concentrations were equal in each treatment (black) or were not quantified (gray). Genes that contain the conserved motif with similarity to the myb-like regulatory element in Arabidopsis thaliana are marked with a red star. The orange and blue letters refer to elevated or depressed transcript levels for each gene (data from Bachy et al. 2018). Descriptions of putative metabolic responses in each pathway are shown. Genes encode for: (A) pyruvate kinase, (B) pyruvate dehydrogenase, (C) citrate synthase, (D) aconitase, (E) $\alpha$-ketoglutarate dehydrogenase, (F) succinate dehydrogenase, $(\mathrm{G})$ fumarase, $(\mathrm{H})$ ribulose-1,5-bisphosphate carboxylase, (I) triose sugar transporter, $(\mathrm{J})$ nucleotide phosphatase. TCA: tricarboxylic acid

specific sequences (the PHO box) where the TF binds to activate or repress the gene (Santos-Beneit 2015). These PHO box sequences are distinct among different bacterial species and strains. In $M$. commoda, the discovered motif was not significantly similar to myb-like DNA-binding motifs in A. thaliana nor was it similar to the motif discovered in $M$. pusilla. Although we expected that the myb domain of the Psr1-like proteins in the 2 Micromonas species would interact with similar binding regions in the genome, psr1-like derived amino acid sequences from M. pusilla and M. commoda were only $47 \%$ similar. TF protein sequences with up to $79 \%$ similarity have been shown to have distinct DNA-binding motif profiles (Franco-Zorrilla et al. 2014), so the 2 proteins in different species could reasonably have distinct DNA-binding motifs. If the Psr1-like TF regulates a different set of genes in the 2 Micromonas species, this may serve as a niche-defining feature between these taxa similar to that described in yeast (Borneman et al. 2007). As further support for this hypothesis, we observed the significant conserved motif only in a subset of genes that were differentially expressed under $\mathrm{P}$ limitation or deficiency (Whitney \& Lomas 2016, Bachy et al. 2018). Both the observed differences in the psr1-like gene and differences in associated binding motifs between the species may represent adaptation to distinct environments, as M. pusilla was collected in the temperate English Channel, while M. commoda was isolated from the tropics (Worden 2006).

The psr1-like gene in M. commoda and M. pusilla was one of the highest DE genes in each experiment (Whitney \& Lomas 2016, Bachy et al. 2018), suggesting that this potential TF plays a critical role in the response of Micromonas to P deficiency. Moreover, the psr1-like gene was highly expressed in 2 culture experiments with different growth conditions and sampled at different growth stages, suggesting a broad physiological relevance for the Psr1-like TF (Fig. 6). In contrast to the transcriptome results, a recent study describing proteome changes in $M$. pusilla CCMP1545 grown under P-limited conditions in a bioreactor (Guo et al. 2018) did not observe sig- 
nificant regulation of the Psr1-like protein (significance defined by Guo et al. 2018). The discordant results may be due to known differences in regulation between genes and proteins (Guo et al. 2018) or to different Psr1-like protein behavior under continuous culturing conditions. Regardless, these results warrant further investigation into the physiological role of the Psr1-like protein in M. pusilla.

The variable and non-uniform dynamics of several TCA cycle metabolites between treatments suggested that this pathway could be involved in the metabolic response to $\mathrm{P}$ deficiency in $M$. pusilla. Indeed, nearly all of the genes involved in the end of glycolysis and in the TCA cycle were up-regulated in $M$. pusilla (Bachy et al. 2018), and these genes contained the significant conserved motif. The gene encoding for fumarase, in particular, was upregulated in both $M$. pusilla and M. commoda transcriptomes under P limitation or deficiency. If fumarase gene expression is regulated by the Psr1-like protein, expression of these 2 genes (fumarase gene and psr1) should be correlated in field populations. We observed support for this hypothesis within the Tara Oceans dataset, but observed weaker relationships between psr1-like gene expression and other up-regulated genes in either $M$. pusilla or M. commoda (Text S3). In M. pusilla, carbon flow through the TCA cycle may increase as a means to fuel triacylglycerol (TAG) or starch production and to pull potentially damaging energy away from the photosystems (Norici et al. 2011, Klok et al. 2013). Similarly, several proteins involved in glycolysis (e.g. pyruvate dehydrogenase) and the TCA cycle (e.g. succinate dehydrogenase) were found to be up-regulated in another study with M. pusilla under P limitation (Guo et al. 2018), lending some support to our hypothesis that carbon flow through the TCA cycle increases under $P$ deficiency. Insight from the latter study underscores potential differences between gene and enzyme regulation in glycolysis and the TCA cycle (Guo et al. 2018). For example, we found congruency in up- or down-regulation of some proteins from Guo et al. (2018) that we would expect in M. pusilla under P deficiency based on our combined comparative genomics and metabolomics approach (i.e. pyruvate kinase, pyruvate dehydrogenase, oxoglutarate dehydrogenase, and succinate dehydrogenase) but not others (i.e. fumarase, aconitase, citrate synthase). Analysis of transcript, protein, and metabolite responses in $M$. pusilla under the same growth conditions will be required to elucidate the role of a Psr1-like TF in this and other taxa.

In addition to TCA cycle intermediates, we observed shifts for several purine nucleosides between treatments and detected the significant conserved motif in genes for a nucleoside phosphatase and aspartate transcarbamylase. These results suggest increased nucleotide salvage through purine nucleotides and nucleosides, a mechanism described in $M$. commoda (Whitney \& Lomas 2016) and in other phytoplankton (Dyhrman \& Palenik 2003, Kujawinski et al. 2017). Previous phytoplankton work has also highlighted expression of the POX-encoding gene (Rokitta et al. 2014, 2016) in nutrient limitation experiments (including $\mathrm{P}$ deficiency) with the haptophyte Emiliania huxleyi, where this enzyme may have a role in stabilizing the mitochondrial membrane and in detecting cellular $\mathrm{N}$ levels. The POX gene was not present in $M$. pusilla but was up-regulated in M. commoda (Whitney \& Lomas 2016; Table S4). Instead, in M. pusilla, a copper amine oxidase was up-regulated (Bachy et al. 2018) and contained the significant conserved motif that may function as a regulatory element for the Psr1-like TF. Thus, POX and copper amine oxidase represent gene targets for further investigation.

Maat et al. (2014) noted that both E. huxleyi (Borchard et al. 2011) and M. pusilla (Maat et al. 2014) exhibited a similar metabolic response to elevated $p \mathrm{CO}_{2}$ levels and P-deficient conditions. While there may be multiple factors underlying the response of these organisms, both contain the psr1-like gene, which could direct part of a shared metabolic response to elevated $p \mathrm{CO}_{2}$ and nutrient-limited conditions. While transcriptomic analysis of $E$. huxleyi was not performed by Borchard et al. (2011), Rokitta et al. (2014, 2016) performed microarray transcriptome analysis of E. huxleyi cells under separate N- and Pdeficient conditions (normal $p \mathrm{CO}_{2}$ ). The transcripts were only partial sequences; however, 2 up-regulated genes detected by Rokitta et al. (2016) contained myb-like DNA binding domains (gene IDs: GJ02872, GJ03978). Targeted experimental work, such as knockout or overexpression analysis (Bajhaiya et al. 2017), is necessary to elucidate the presence and role of a psr1-like gene in E. huxleyi. If there is a common role for the psr1-like gene in $E$. huxleyi and $M$. pusilla in their response to nutrient limitation and elevated $p \mathrm{CO}_{2}$, this could have important implications for predicting the physiological response of the wide range of organisms that contain this psr1-like gene to changing environmental conditions.

The potential impact of Psr1-like regulated genes on the ecological roles of Micromonas spp. is unknown, but our results suggest that the Psr1-like protein coordinates a metabolic shift in these organisms under $\mathrm{P}$ 
deficiency, altering the intracellular flow of carbon and other elements. More comprehensive examination of these metabolic responses, which likely vary to some extent among organisms, will be paramount to improving models of trophic carbon flow. More experiments are needed to characterize the structure and role of the psr1-like gene in Micromonas spp. and other phytoplankton, including: (1) confirming the presence of the $p s r 1$-like gene with genetic experiments, (2) determining the conditions under which the Psr1-like protein is abundant, (3) identifying the taxon-specific genes affected by the Psr1-like protein, (4) verifying the interaction of the Psr1-like protein with the hypothesized binding sites, and (5) comparing the genetic and metabolic responses to $\mathrm{P}$ deficiency between organisms containing the psr1-like gene and those that do not. Exploring the underlying biology of the psr1-like gene will facilitate mechanistic understanding of the complex metabolic response of these organisms to P limitation and will enhance our ecological and biogeochemical predictions.

Acknowledgements. We thank Krista Longnecker for performing the total organic carbon analysis and Matthew Johnson and Elizabeth Harvey for assistance with flow cytometry and fluorescence induction and relaxation analysis. This research was funded by the Gordon and Betty Moore Foundation through Grant GBMF3304 to E.B.K., and it was partially supported by a grant from the Simons Foundation (Award ID 509034 to E.B.K.).

\section{LITERATURE CITED}

Alipanah L, Winge P, Rohloff J, Najafi J, Brembu T, Bones AM (2018) Molecular adaptations to phosphorus deprivation and comparison with nitrogen deprivation responses in the diatom Phaeodactylum tricornutum. PLOS ONE 13(2):e0193335

Bachy C, Charlesworth CJ, Chan AM, Finke JF and others (2018) Transcriptional responses of the marine green alga Micromonas pusilla and an infecting prasinovirus under different phosphate conditions. Environ Microbiol 20:2898-2912

Bailey TL, Boden M, Buske FA, Frith M and others (2009) MEME Suite: tools for motif discovery and searching. Nucl Acid Res 37:W202-W208

Bajhaiya AK, Moreira JZ, Pittman JK (2017) Transcriptional engineering of microalgae: prospects for high-value chemicals. Trends Biotechnol 35:95-99

Barrett LW, Fletcher S, Wilton SD (2012) Regulation of eukaryotic gene expression by the untranslated gene regions and other non-coding elements. Cell Mol Life Sci 69:3613-3634

* Benjamini Y, Hochberg Y (1995) Controlling the false discovery rate: a practical and powerful approach to multiple testing. J R Statist Soc B 57: 289-300

Berdalet E, Latasa M, Estrada M (1994) Effects of nitrogen and phosphorus starvation on nucleic acid and protein content of Heterocapsa sp. J Plankton Res 16:303-316
Borchard C, Borges AV, Haendel N, Engel A (2011) Biogeochemical response of Emiliania huxleyi (PML B92/11) to elevated $\mathrm{CO}_{2}$ and temperature under phosphorous [sic] limitation: a chemostat study. J Exp Mar Biol Ecol 410: 61-71

Borneman AR, Gianoulis TA, Zhang ZD, Yu H and others (2007) Divergence of transcription factor binding sites across related yeast species. Science 317:815-819

Cañavate JP, Armada I, Hachero-Cruzado I (2017) Common and species-specific effects of phosphate on marine microalgae fatty acids shape their function in phytoplankton trophic ecology. Microb Ecol 74:623-639

Carradec Q, Pelletier E, Da Silva C, Alberti A and others (2018) A global ocean atlas of eukaryotic genes. Nat Commun 9:373

Chung CC, Hwang SPL, Chang J (2003) Identification of a high-affinity phosphate transporter gene in a prasinophyte alga, Tetraselmis chui, and its expression under nutrient limitation. Appl Environ Microbiol 69:754-759

* De Carvalho CCCR, Fernandes P (2010) Production of metabolites as bacterial responses to the marine environment. Mar Drugs 8:705-727

* Demory D, Arsenieff L, Simon N, Six C and others (2017) Temperature is a key factor in Micromonas-virus interactions. ISME J 11:601-612

* Derelle E, Ferraz C, Rombauts S, Rouzé P and others (2006) Genome analysis of the smallest free-living eukaryote Ostreococcus tauri unveils many unique features. Proc Natl Acad Sci USA 103:11647-11652

* Dittmar T, Koch B, Hertkorn N, Kattner G (2008) A simple and efficient method for the solid-phase extraction of dissolved organic matter (SPE-DOM) from seawater. Limnol Oceanogr Methods 6:230-235

* Dyhrman ST, Palenik B (2003) Characterization of ectoenzyme activity and phosphate-regulated proteins in the coccolithophorid Emiliania huxleyi. J Plankton Res 25:1215-1225

*Dyhrman ST, Ruttenberg KC (2006) Presence and regulation of alkaline phosphatase activity in eukaryotic phytoplankton from the coastal ocean: implications for dissolved organic phosphorus remineralization. Limnol Oceanogr 51:1381-1390

*Dyhrman ST, Benitez-Nelson CR, Orchard ED, Haley ST, Pellechia PJ (2009) A microbial source of phosphonates in oligotrophic marine systems. Nat Geosci 2:696-699

* Dyhrman ST, Jenkins DB, Rynearson TA, Saito MA and others (2012) The transcriptome and proteome of the diatom Thalassiosira pseudonana reveal a diverse phosphorus stress response. PLOS ONE 7:e33768

*Edgar RC (2004) MUSCLE: multiple sequence alignment with high accuracy and high throughput. Nucleic Acids Res 32:1792-1797

*Elser JJ, Bracken MES, Cleland EE, Gruner DS and others (2007) Global analysis of nitrogen and phosphorus limitation of primary producers in freshwater, marine and terrestrial ecosystems. Ecol Lett 10:1135-1142

Felsenstein J (1981) Evolutionary trees from DNA sequences: a maximum likelihood approach. J Mol Evol 17: 368-376

Feng TY, Yan ZK, Zheng JW, Xie Y and others (2015) Examination of metabolic responses to phosphorus limitation via proteomic analyses in the marine diatom Phaeodactylum tricornutum. Sci Rep 5:10373

Fiore CL, Longnecker K, Kido Soule MC, Kujawinski EB (2015) Release of ecologically relevant metabolites by 
the cyanobacterium Synechococcus elongatus CCMP 1631. Environ Microbiol 17:3949-3963

Franco-Zorrilla JM, López-Vidriero I, Carrasco JL, Godoy M, Vera P, Solano R (2014) DNA-binding specificities of plant transcription factors and their potential to define target genes. Proc Natl Acad Sci USA 111:2367-2372

Goncalves EC, Johnson JV, Rathinasabapathi B (2013) Conversion of membrane lipid acyl groups to triacylglycerol and formation of lipid bodies upon nitrogen starvation in biofuel green algae Chlorella UTEX29. Planta 238: 895-906

Guo J, Wilken S, Jimenez V, Choi CJ and others (2018) Specialized proteomic responses and an ancient photoprotection mechanism sustain marine green algal growth during phosphate limitation. Nat Microbiol 3:781-790

Gupta S, Stamatoyannopolous JA, Bailey T, Noble WS (2007) Quantifying similarity between motifs. Genome Biol 8:R24

Halsey KH, Milligan AJ, Behrenfeld MJ (2014) Contrasting strategies of photosynthetic energy utilization drive lifestyle strategies in ecologically important picoeukaryotes. Metabolites 4:260-280

Hockin NL, Mock T, Mulholland F, Kopriva S, Malin G (2012) The response of diatom central carbon metabolism to nitrogen starvation is different from that of green algae and higher plants. Plant Physiol 158:299-312

* Hoppe CJM, Flintrop CM, Rost B (2018) The Arctic picoeukaryote Micromonas pusilla benefits synergistically from warming and ocean acidification. Biogeosciences 15:4353-4365

Johnson LK, Alexander H, Brown CT (2019) Re-assembly, quality evaluation, and annotation of 678 microbial eukaryotic reference transcriptomes. GigaScience 8: giy158

Johnson WM, Kido Soule MC, Kujawinski EB (2017) Extraction efficiency and quantification of dissolved metabolites in targeted marine metabolomics. Limnol Oceanogr Methods 15:417-428

Jones DT, Taylor WR, Thornton JM (1992) The rapid generation of mutation data matrices from protein sequences. Comput Appl Biosci 8:275-282

Karl DM (2014) Microbially mediated transformations of phosphorus in the sea: new views of an old cycle. Annu Rev Mar Sci 6:279-337

Kido Soule MCK, Longnecker K, Johnson WM, Kujawinski EB (2015) Environmental metabolomics: analytical strategies. Mar Chem 177:374-387

Kishino H, Miyata T, Hasegawa M (1990) Maximum likelihood inference of protein phylogeny and the origin of chloroplasts. J Mol Evol 31:151-160

Klok AJ, Martens DE, Wijffels RH, Lamers PP (2013) Simultaneous growth and neutral lipid accumulation in microalgae. Bioresour Technol 134:233-243

Kujawinski EB, Longnecker K, Alexander H, Dyhrman ST, Fiore CL, Haley ST, Johnson WM (2017) Phosphorus availability regulates intracellular nucleotides in marine eukaryotic phytoplankton. Limnol Oceanogr Lett 2: 119-129

Kumar S, Stecher G, Tamura K (2016) MEGA7: Molecular Evolutionary Genetics Analysis version 7.0 for bigger datasets. Mol Biol Evol 33:1870-1874

Lai J, Yu Z, Song X, Cao X, Han X (2011) Responses of the growth and biochemical composition of Prorocentrum donghaiense to different nitrogen and phosphorus concentrations. J Exp Mar Biol Ecol 405:6-17
Li M, Shi X, Guo C, Lin S (2016) Phosphorus deficiency inhibits cell division but not growth in the dinoflagellate Amphidinium carterae. Front Microbiol 7:826

* Li WKW (1994) Primary production of prochlorophytes, cyanobacteria, and eukaryotic ultraphytoplankton: measurements from flow cytometric sorting. Limnol Oceanogr 39:169-175

Lin S, Litaker RW, Sundra WG (2016) Phosphorus physiological ecology and molecular mechanisms in marine phytoplankton. J Phycol 52:10-36

Koebl M, Cockshutt AM, Campbell D, Finkel ZV (2010) Physiological basis for high resistance to photoinhibition under nitrogen depletion in Emiliania huxleyi. Limnol Oceanogr 55:2150-2160

KLomas MW, Swain A, Shelton R, Ammerman JW (2004) Taxonomic variability of phosphorus stress in Sargasso Sea phytoplankton. Limnol Oceanogr 49:2303-2309

KLongnecker K (2015) Dissolved organic matter in newly formed sea ice and surface seawater. Geochim Cosmochim Acta 171:39-49

*Maat DS, Crawfurd KJ, Timmermans KR, Brussaard CPD (2014) Elevated $\mathrm{CO}_{2}$ and phosphate limitation favor Micromonas pusilla through stimulated growth and reduced viral impact. Appl Environ Microbiol 80: 3119-3127

Maat DS, van Bleijswijk JDL, Witte HJ, Brussaard PD (2016) Virus production in phosphorus-limited Micromonas pusilla stimulated by a supply of naturally low concentrations of different phosphorus sources, far into the lytic cycle. FEMS Microbiol Ecol 92:fiw136

* Marchler-Bauer A, Bryant SH (2004) CD-Search: protein domain annotations on the fly. Nucleic Acids Res 32(Suppl 2):W327-W331

Markou G, Nerantzis E (2013) Microalgae for high-value compounds and biofuels production: a review with focus on cultivation under stress conditions. Biotechnol Adv 31:1532-1542

Martin P, Van Mooy BAS, Heithoff A, Dyhrman ST (2011) Phosphorus supply drives rapid turnover of membrane phospholipids in the diatom Thalassiosira pseudonana. ISME J 5:1057-1060

Martin P, Dyhrman ST, Lomas MW, Poulton NJ, Van Mooy BAS (2014) Accumulation and enhanced cycling of polyphosphate by Sargasso Sea phytoplankton in response to low phosphorus. Proc Natl Acad Sci USA 111:8089-8094

* Martiny AC, Ustick L, Garcia CA, Lomas MW (2020) Genomic adaptation of marine phytoplankton populations regulates phosphate uptake. Limnol Oceanogr 65:S340-S350

*Moseley JL, Chang CW, Grossman AR (2006) Genomebased approaches to understanding phosphorus deprivation responses and PSR1 control in Chlamydomonas reinhardtii. Eukaryot Cell 5:26-44

* Müller R, Morant M, Jarmer H, Nilsson L, Nielsen TH (2007) Genome-wide analysis of the Arabidopsis leaf transcriptome reveals interaction of phosphate and sugar metabolism. Plant Physiol 143:156-171

Norici A, Bazonni AM, Pugnetti A, Raven JA, Giordano M (2011) Impact of irradiance on the $C$ allocation in the coastal marine diatom Skeletonema marinoi Sarno and Zingone. Plant Cell Environ 34:1666-1677

Rengefors K, Ruttenberg KC, Haupert CL, Taylor C, Howes BL, Anderson DM (2003) Experimental investigation of taxon-specific response of alkaline phosphatase activity in natural freshwater phytoplankton. Limnol Oceanogr 48:1167-1175 
Rokitta SD, von Dassow P, Rost B, John U (2014) Emiliania huxleyi endures $\mathrm{N}$-limitation with an efficient metabolic budgeting and effective ATP synthesis. BMC Genomics 15:1051

Rokitta SD, von Dassow P, Rost B, John U (2016) P- and Ndepletion trigger similar cellular responses to promote senescence in eukaryotic phytoplankton. Front Mar Sci 3:109

Rubio V, Linhares F, Solano R, Martín AC, Iglesias J, Leyva A, Paz-Ares J (2001) A conserved MYB transcription factor involved in phosphate starvation signaling both in vascular plants and in unicellular algae. Genes Dev 15: 2122-2133

Rusch DB, Halpern AL, Sutton G, Heidelberg KB and others (2007) The Sorcerer II global ocean sampling expedition: northwest Atlantic through eastern tropical Pacific. PLOS Biol 5:e77

Santos-Beneit F (2015) The Pho regulon: a huge regulatory network in bacteria. Front Microbiol 6:402

Shemi A, Schatz D, Fredricks HF, Van Mooy BAS, Porat Z, Vardi A (2016) Phosphorus starvation induces membrane remodeling and recycling in Emiliania huxleyi. New Phytol 211:886-898

Shi X, Lin X, Li L, Palenik B, Lin S (2017) Transcriptomic and microRNAomic profiling reveals multi-faceted mecha-

Editorial responsibility: Zoe Finkel,

Sackville, New Brunswick, Canada

Reviewed by: 3 anonymous referees nisms to cope with phosphate stress in a dinoflagellate. ISME J 11:2209-2218

Sosa OA, Caey JR, Karl DM (2019) Methylphosphonate oxidation in Prochlorococcus strain MIT9301 supports phosphate acquisition, formate excretion and carbon assimilation into purines. Appl Environ Microbiol 85: e00289-19

Tyrrell $\mathrm{T}$ (1999) The relative influences of nitrogen and phosphorus on oceanic primary production. Nature 400: 525-531

* Van Mooy BAS, Fredricks HF, Pedler BE, Dyhrman ST and others (2009) Phytoplankton in the ocean use nonphosphorus lipids in response to phosphorus scarcity. Nature 458:69-72

*Whitney LP, Lomas MW (2016) Growth on ATP elicits a Pstress response in the picoeukaryote Micromonas pusilla. PLOS ONE 11:e0155158

Worden AZ (2006) Picoeukaryote diversity in coastal waters of the Pacific Ocean. Aquat Microb Ecol 43: 165-175

WWykoff DD, Grossman AR, Weeks DP, Usuda H, Shimogawara K (1999) Psr1, a nuclear localized protein that regulates phosphorus metabolism in Chlamydomonas. Proc Natl Acad Sci USA 96:15336-15341

Submitted: October 16, 2019

Accepted: November 10, 2020

Proofs received from author(s): February 11, 2021 